\title{
Introduction of new Picard-S hybrid iteration with application and some results for nonexpansive mappings
}

New Picard-S hybrid iteration

\author{
Julee Srivastava \\ Department of Mathematics and Statistics, \\ Deen Dayal Upadhyaya Gorakhpur University, Gorakhpur, India
}

Received 24 August 2020 Revised 5 November 2020 29 November 2020 Accepted 1 December 2020

\begin{abstract}
Purpose - In this paper, Picard-S hybrid iterative process is defined, which is a hybrid of Picard and S-iterative process. This new iteration converges faster than all of Picard, Krasnoselskii, Mann, Ishikawa, S-iteration, Picard-Mann hybrid, Picard-Krasnoselskii hybrid and Picard-Ishikawa hybrid iterative processes for contraction mappings and to find the solution of delay differential equation, using this hybrid iteration also proved some results for Picard-S hybrid iterative process for nonexpansive mappings.

Design/methodology/approach - This new iteration converges faster than all of Picard, Krasnoselskii, Mann, Ishikawa, S-iteration, Picard-Mann hybrid, Picard-Krasnoselskii hybrid, Picard-Ishikawa hybrid iterative processes for contraction mappings.

Findings - Showed the fastest convergence of this new iteration and then other iteration defined in this paper. The author finds the solution of delay differential equation using this hybrid iteration. For new iteration, the author also proved a theorem for nonexpansive mapping.

Originality/value - This new iteration converges faster than all of Picard, Krasnoselskii, Mann, Ishikawa, S-iteration, Picard-Mann hybrid, Picard-Krasnoselskii hybrid, Picard-Ishikawa hybrid iterative processes for contraction mappings and to find the solution of delay differential equation, using this hybrid iteration also proved some results for Picard-S hybrid iterative process for nonexpansive mappings.
\end{abstract}

Keywords Fixed point, Iteration, Contraction, Nonexpansive mappings

Paper type Research paper

\section{Introduction}

Let $E$ be a normed linear space and $C$ be a non-empty convex subset of $E$. A mapping $T: C \rightarrow C$ is called contraction if

$$
\|T x-T y\| \leq \delta\|x-y\|
$$

for all $x, y \in C$ and $\delta \in(0,1)$.

Let $C$ be a non-empty subset of a normed linear space $E$ and $T: C \rightarrow E$ a mapping. Then $T$ is said to be nonexpansive if

$$
\|T x-T y\| \leq\|x-y\|, \text { for all } x, y \in C
$$

A sequence $x_{n} \subset C$ is an approximating fixed point sequence of $T$ if $\lim _{n \rightarrow \infty}\left\|x_{n}-T x_{n}\right\|=0$. We say that $x \in C$ is a fixed point of $T$ if $T(x)=x$ and denote $F(T)$ the set of all fixed points of $T$.

\section{JEL Classification - 47H09, 47H10, 54H25, 47J25}

(C) Julee Srivastava. Published in Arab Journal of Mathematical Sciences. Published by Emerald Publishing Limited. This article is published under the Creative Commons Attribution (CC BY 4.0) licence. Anyone may reproduce, distribute, translate and create derivative works of this article (for both commercial and non-commercial purposes), subject to full attribution to the original publication and authors. The full terms of this licence may be seen at http://creativecommons.org/licences/by/4.0/legalcode

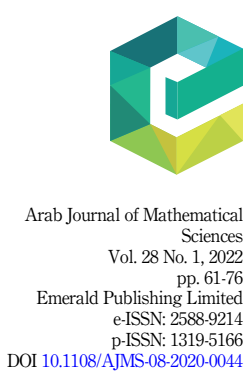


AJMS

28,1

In this paper, $N$ denotes the set of all positive integers.

The Picard iterative process [1] is defined by the sequence $\left\{u_{n}\right\}$ as follows:

$$
\begin{aligned}
u_{1} & =u \in C \\
u_{n+1} & =T u_{n}, \quad n \in N
\end{aligned}
$$

The Krasnoselskii iterative process [2] is defined by the sequence $\left\{v_{n}\right\}$ :

$$
\begin{aligned}
v_{1} & =v \in C \\
v_{n+1} & =(1-\lambda) v_{n}+\lambda T v_{n}, \quad n \in N
\end{aligned}
$$

where $\lambda \in(0,1)$.

The Mann iteration [3] is defined by the sequence $\left\{w_{n}\right\}$ :

$$
\begin{aligned}
w_{1} & =w \in C \\
w_{n+1} & =\left(1-\alpha_{n}\right) w_{n}+\alpha_{n} T w_{n}, \quad n \in N
\end{aligned}
$$

where $\left\{\alpha_{n}\right\} \subset(0,1)$ satisfies certain appropriate conditions.

The Ishikawa iterative process [4] is defined by the sequence $\left\{z_{n}\right\}$ :

$$
\begin{aligned}
z_{1} & =z \in C \\
z_{n+1} & =\left(1-\alpha_{n}\right) z_{n}+\alpha_{n} T y_{n} \\
y_{n} & =\left(1-\beta_{n}\right) z_{n}+\beta_{n} T z_{n}, \quad n \in N
\end{aligned}
$$

where $\left\{\alpha_{n}\right\},\left\{\beta_{n}\right\} \subset(0,1)$ satisfies certain appropriate conditions.

S-iterative process [5] is defined by the sequence $\left\{q_{n}\right\}$ :

$$
\begin{aligned}
q_{1} & =q \in C \\
q_{n+1} & =\left(1-\alpha_{n}\right) T q_{n}+\alpha_{n} T y_{n} \\
y_{n} & =\left(1-\beta_{n}\right) q_{n}+\beta_{n} T q_{n}, \quad n \in N
\end{aligned}
$$

where $\left\{\alpha_{n}\right\},\left\{\beta_{n}\right\} \subset(0,1)$ satisfies certain appropriate conditions.

Many important non-linear problems of applied mathematics are usually constructed in the form of fixed point equation. These problems are related with physical problem of applied sciences and engineering.

The Picard iteration is the simple iteration for approximate solution of fixed point equation for non-linear contraction mapping. Some results based on Picard iteration are introduced by Chidume and Olaleru [6]. $\left\{s_{n}\right\}$

Khan [7] introduced the Picard-Mann hybrid iterative process defined by the sequence

$$
\begin{aligned}
s_{1} & =s \in C \\
s_{n+1} & =T y_{n} \\
y_{n} & =\left(1-\alpha_{n}\right) s_{n}+\alpha_{n} T s_{n}, \quad n \in N
\end{aligned}
$$

where $\left\{\alpha_{n}\right\}$ is a real sequence in $(0,1)$.

Okeke and Abbas [8] introduced the Picard-Krasnoselskii hybrid iterative process defined by the sequence $\left\{m_{n}\right\}$ :

$$
\begin{aligned}
m_{1} & =m \in C \\
m_{n+1} & =T y_{n} \\
y_{n} & =(1-\lambda) m_{n}+\lambda T m_{n}, \quad n \in N
\end{aligned}
$$

where $\lambda \in(0,1)$. 
Okeke [9] introduced the Picard-Ishikawa hybrid iterative process defined by the sequence $\left\{t_{n}\right\}$ :

$$
\begin{aligned}
t_{1} & =t \in C \\
t_{n+1} & =T v_{n} v \\
v_{n} & =\left(1-\alpha_{n}\right) t_{n}+\alpha_{n} T u_{n} \\
u_{n} & =\left(1-\beta_{n}\right) t_{n}+\beta_{n} T t_{n}, \quad n \in N
\end{aligned}
$$

where $\left\{\alpha_{n}\right\},\left\{\beta_{n}\right\}$ are real sequences in $(0,1)$. Using hybridization with Picard, now I introduce

Picard-S hybrid iterative process defined by the sequence $\left\{x_{n}\right\}$ :

$$
\begin{aligned}
x_{1} & =x \in C \\
x_{n+1} & =T z_{n} \\
z_{n} & =\left(1-\alpha_{n}\right) T x_{n}+\alpha_{n} T y_{n} \\
y_{n} & =\left(1-\beta_{n}\right) x_{n}+\beta_{n} T x_{n}, \quad n \in N
\end{aligned}
$$

where $\left\{\alpha_{n}\right\}$ and $\left\{\beta_{n}\right\}$ are real sequences in $(0,1)$ satisfying condition:

$$
\sum_{n=1}^{\infty} \alpha_{n} \beta_{n}\left(1-\beta_{n}\right)=\infty
$$

Let $\left\{u_{n}\right\}$ and $\left\{v_{n}\right\}$ be two fixed point iteration processes that converge to a certain fixed point $p$ of a given operator $T$. The sequence $\left\{u_{n}\right\}$ is better than $\left\{v_{n}\right\}$ if

$$
\left\|u_{n}-p\right\| \leq\left\|v_{n}-p\right\|
$$

for all $n \in N$ (given by Rhodes [10]).

\section{Preliminaries}

Definition 2.1. Let $\left\{a_{n}\right\}$ and $\left\{b_{n}\right\}$ be two sequences of real numbers converging to $a$ and $b$, respectively. If

$$
\lim _{n \rightarrow \infty} \frac{\left|a_{n}-a\right|}{\left|b_{n}-b\right|}=0
$$

then $\left\{a_{n}\right\}$ converges faster than $\left\{b_{n}\right\}$.

Definition 2.2. Let $\left\{u_{n}\right\}$ and $\left\{v_{n}\right\}$ be two fixed point iterative processes, both converge to fixed point $p$ of a given operator $T$. Suppose that the error estimates.

$$
\begin{aligned}
& \left\|u_{n}-p\right\| \leq a_{n}, \quad \text { for all } n \in N \\
& \left\|v_{n}-p\right\| \leq b_{n}, \quad \text { for all } n \in N
\end{aligned}
$$

are available, where $\left\{a_{n}\right\}$ and $\left\{b_{n}\right\}$ are two sequences of positive numbers converging to 0 . If $\left\{a_{n}\right\}$ converges faster than $\left\{b_{n}\right\}$, then $\left\{u_{n}\right\}$ converges faster than $\left\{v_{n}\right\}$ to $p$.

Definition 2.3. Let $X$ be a Banach space. Then a function $\delta_{X}:[0,2] \rightarrow[0,1]$ is said to be the modulus of convexity of $X$ if

$$
\delta_{X}(\epsilon)=\inf \left\{1-\left\|\frac{x+y}{2}\right\|:\|x\| \leq 1,\|y\| \leq 1,\|x-y\| \geq \epsilon\right\}
$$

It is easy to see that $\delta_{X}(0)=0$ and $\delta_{X}(t) \geq 0$ for all $t \geq 0$. References [10-19] dealing with rate of convergence of iterative process. Some authors analyse its stability. We need following lemma to prove result. 
AJMS

28,1

Lemma 2.1. Let $\left\{s_{n}\right\}$ be a sequence of positive real numbers which satisfies

$$
\begin{gathered}
s_{n+1} \leq\left(1-\mu_{n}\right) s_{n} \\
\text { If }\left\{\mu_{n}\right\} \subset(0,1) \text { and } \sum_{n=1}^{\infty} \mu_{n}=\infty, \text { then } \lim _{n \rightarrow \infty} s_{n}=0 .
\end{gathered}
$$

The aim of this paper is to introduce the Picard-S hybrid iterative process and to show that this new iterative process is faster than all of Picard, Krasnoselskii, Mann, Ishikawa in sense of Berinde [20], S-iteration in sense of Agarwal [5], Picard-Mann hybrid in sense of Khan [7], Picard-Krasnoselskii hybrid in sense of Okeke [8] and Picard-Ishikawa hybrid in the sense of Okeke [9].

Okeke already proved that Picard-Krasnoselskii hybrid iterative process converges faster than Picard, Krasnoselskii, Mann and Ishikawa. Khan [7] proved that Picard-Mann hybrid iterative process converges faster than Picard, Mann, Ishikawa iterative processes. Therefore, I show that my new Picard-S hybrid iterative process converges faster than S-iteration, PicardMann hybrid iteration, Picard-Krasnoselskii hybrid iteration and Picard-Ishikawa hybrid iterative process in the topic Rate of Convergence. In 2020, Zhao [21] proved existence and uniqueness of pseudo almost periodic solution for a class of iterative functional differential equations with delays depending on state. In next section, I find the solution of delay differential equation using Picard-S hybrid iterative process. Aynur Sahin [22] proved some strong convergence results of Picard-Krasnoselskii hybrid iterative process for a general class of contractive-like operator in hyperbolic space. In next section, I prove some results of Picard-S hybrid iterative process for nonexpansive mappings in uniformly convex Banach space.

\section{Rate of convergence}

Proposition 3.1. Let $C$ be a non-empty closed convex subset of a normed space $E$ and let $T$ be a contraction of $C$ into itself. Suppose that each of the iterative process 1.6, 1.7, 1.8, 1.9 and 1.10 converges to the same fixed point $p$ of $T$ where $\left\{\alpha_{n}\right\}$ and $\left\{\beta_{n}\right\}$ are sequences in $(0.1)$ such that $0<\lambda \leq \alpha_{n}, \beta_{n}<1$ for all $n \in N$ and for some $\lambda$ and $\delta \in(0,1)$ is a Lipschitz constant for contraction mapping $T$. Then Picard-S hybrid iterative process defined by (1.10) converges faster than all the other four iterations.

Proof: Suppose that $p$ is the fixed point of the operator $T$. Using (1.1) and S-iterative process (1.6), we have

$$
\begin{aligned}
\left\|q_{n+1}-p\right\| & \leq\left(1-\alpha_{n}\right) \delta\left\|q_{n}-p\right\|+\alpha_{n} \delta\left\|y_{n}-p\right\| \\
& \leq \delta\left[\left(1-\alpha_{n}\right)\left\|q_{n}-p\right\|+\alpha_{n}\left\{\left(1-\beta_{n}\right)\left\|q_{n}-p\right\|+\delta \beta_{n}\left\|q_{n}-p\right\|\right\}\right] \\
& =\delta\left[1-(1-\delta) \alpha_{n} \beta_{n}\right]\left\|q_{n}-p\right\| \\
& =\left[\delta-\delta \alpha_{n} \beta_{n}+\delta^{2} \alpha_{n} \beta_{n}\right]\left\|q_{n}-p\right\| \\
& \leq\left[\delta-\delta \alpha_{n} \beta_{n}+\delta \alpha_{n} \beta_{n}\right]\left\|q_{n}-p\right\| \\
& =\delta\left\|q_{n}-p\right\| \\
& \leq \delta^{2}\left\|q_{n-1}-p\right\| \\
& \vdots \\
& \leq \delta^{n}\left\|q_{1}-p\right\| \\
\text { Let } a_{n} & =\delta^{n}\left\|q_{1}-p\right\|
\end{aligned}
$$

Now using (1.1) and Picard-Mann hybrid iterative process (1.7), we have 


$$
\begin{aligned}
\left\|s_{n+1}-P\right\| & =\left\|T y_{n}-p\right\| \\
& \leq \delta\left\|y_{n}-p\right\| \\
& =\delta\left[\left(1-\alpha_{n}\right)\left\|s_{n}-p\right\|+\alpha_{n}\left\|T s_{n}-p\right\|\right] \\
& \leq \delta\left[\left(1-\alpha_{n}\right)\left\|s_{n}-p\right\|+\alpha_{n} \delta\left\|s_{n}-p\right\|\right] \\
& =\delta\left[\left(1-\alpha_{n}+\alpha_{n} \delta\right)\left\|s_{n}-p\right\|\right] \\
& =\delta\left(1-(1-\delta) \alpha_{n}\right)\left\|s_{n}-p\right\| \\
& \leq \delta\left(1-(1-\delta) \lambda^{2}\right)\left\|s_{n}-p\right\| \\
& \vdots \\
& \leq\left[\delta\left(1-(1-\delta) \lambda^{2}\right)\right]^{n}\left\|s_{1}-p\right\| \\
\text { Let } \quad b_{n} & =\left[\delta\left(1-(1-\delta) \lambda^{2}\right)\right]^{n}\left\|s_{1}-p\right\|
\end{aligned}
$$

New Picard-S hybrid iteration

Using (1.1) and Picard-Krasnoselskii hybrid iteration (1.8)

$$
\begin{aligned}
\left\|m_{n+1}-p\right\| & =\left\|T y_{n}-p\right\| \\
& \leq \delta\left\|y_{n}-p\right\| \\
& \leq \delta\left\|(1-\lambda)\left(m_{n}-p\right)+\lambda\left(T m_{n}-p\right)\right\| \\
& \leq \delta\left[(1-\lambda)\left\|m_{n}-p\right\|+\lambda \delta\left\|m_{n}-p\right\|\right] \\
& =\delta\left[\left(1-(1-\delta) \lambda^{2}\right)\right]\left\|m_{n}-p\right\| \\
& \vdots \\
& \leq\left[\delta\left(1-(1-\delta) \lambda^{2}\right)\right]^{n}\left\|m_{1}-p\right\| \\
\text { Let } \quad c_{n} & =\left[\delta\left(1-(1-\delta) \lambda^{2}\right)\right]^{n}\left\|m_{1}-p\right\|
\end{aligned}
$$

Using (1.1) and Picard-Ishikawa hybrid iterative process (1.9), we have

$$
\begin{aligned}
\left\|t_{n+1}-p\right\| & =\left\|T v_{n}-p\right\| \\
& \leq \delta\left\|v_{n}-p\right\| \\
\left\|u_{n}-p\right\| & =\left\|\left(1-\beta_{n}\right) t_{n}+\beta_{n} T t_{n}-p\right\| \\
& \leq\left(1-\beta_{n}\right)\left\|t_{n}-p\right\|+\beta_{n}\left\|T t_{n}-p\right\| \\
& \leq\left(1-\beta_{n}\right)\left\|t_{n}-p\right\|+\beta_{n} \delta\left\|t_{n}-p\right\| \\
& =\left(1-\beta_{n}+\beta_{n} \delta\right)\left\|t_{n}-p\right\| \\
& =\left[1-\beta_{n}(1-\delta)\right]\left\|t_{n}-p\right\| \\
\left\|v_{n}-p\right\| & =\left\|\left(1-\alpha_{n}\right) t_{n}+\alpha_{n} T u_{n}-p\right\| \\
& \leq\left(1-\alpha_{n}\right)\left\|t_{n}-p\right\|+\alpha_{n}\left\|T u_{n}-p\right\| \\
& \leq\left(1-\alpha_{n}\right)\left\|t_{n}-p\right\|+\alpha_{n} \delta\left\|u_{n}-p\right\| \\
& \leq\left(1-\alpha_{n}\right)\left\|t_{n}-p\right\|+\alpha_{n} \delta\left[1-\beta_{n}(1-\delta)\right]\left\|t_{n}-p\right\| \\
& =\left[1-\alpha_{n}+\alpha_{n} \delta\left\{1-\beta_{n}(1-\delta)\right\}\right]\left\|t_{n}-p\right\| \\
& =\left[1-\alpha_{n}+\alpha_{n} \delta-\alpha_{n} \beta_{n}(1-\delta)\right]\left\|t_{n}-p\right\| \\
& =\left[1-\alpha_{n}(1-\delta)-\alpha_{n} \beta_{n}(1-\delta)\right]\left\|t_{n}-p\right\| \\
& \leq\left[1-\alpha_{n}(1-\delta)\right]\left\|t_{n}-p\right\|
\end{aligned}
$$

Now, $\quad\left\|t_{n+1}-p\right\| \leq \delta\left[1-\alpha_{n}(1-\delta)\right]\left\|t_{n}-p\right\|$

$$
\leq \delta\left[1-\lambda^{2}(1-\delta)\right]\left\|t_{n}-p\right\|
$$$$
\begin{aligned}
& \leq \delta^{n}\left[1-\lambda^{2}(1-\delta)\right]^{n}\left\|t_{1}-p\right\| \\
\text { Let } \quad d_{n} & =\delta^{n}\left[1-\lambda^{2}(1-\delta)\right]^{n}\left\|t_{1}-p\right\|
\end{aligned}
$$

Using (1.1) and Picard-S hybrid iterative process (1.10), we have 
AJMS

28,1

66

$$
\begin{aligned}
\left\|x_{n+1}-p\right\| & =\left\|T z_{n}-p\right\| \\
& \leq \delta\left\|z_{n}-p\right\| \\
& \leq \delta\left\|\left(1-\alpha_{n}\right) T x_{n}+\alpha_{n} T y_{n}-p\right\| \\
& \leq \delta\left\|\left(1-\alpha_{n}\right)\left(T x_{n}-p\right)+\alpha_{n}\left(T y_{n}-p\right)\right\| \\
& \leq \delta^{2}\left(1-\alpha_{n}\right)\left\|x_{n}-p\right\|+\delta^{2} \alpha_{n}\left\|y_{n}-p\right\| \\
& =\delta^{2}\left[\left(1-\alpha_{n}\right)\left\|x_{n}-p\right\|+\alpha_{n}\left\{\left(1-\beta_{n}\right)\left\|x_{n}-p\right\|+\beta_{n} \delta\left\|x_{n}-p\right\|\right\}\right] \\
& =\delta^{2}\left[1-\alpha_{n}+\alpha_{n}\left(1-\beta_{n}\right)+\alpha_{n} \beta_{n} \delta\right]\left\|x_{n}-p\right\| \\
& =\delta^{2}\left[1-(1-\delta) \alpha_{n} \beta_{n}\right]\left\|x_{n}-p\right\| \\
& \leq \delta^{2}\left[1-(1-\delta) \lambda^{2}\right]\left\|x_{n}-p\right\| \\
& \vdots \\
\text { Let } e_{n} & \leq\left[\delta^{2}\left(1-(1-\delta) \lambda^{2}\right)\right]^{n}\left\|x_{1}-p\right\| \\
& {\left[\delta^{2}\left(1-(1-\delta) \lambda^{2}\right)\right]^{n}\left\|x_{1}-p\right\| }
\end{aligned}
$$

Now compute the rate of convergence of Picard-S iterative process (1.10) as follows:

$$
\text { (i) } \begin{aligned}
\frac{e_{n}}{a_{n}} & =\frac{\left[\delta^{2}\left(1-(1-\delta) \lambda^{2}\right)\right]^{n}}{\delta^{n}\left\|q_{1}-p\right\|}\left\|x_{1}-p\right\| \\
& =\delta^{n}\left(1-(1-\delta) \lambda^{2}\right)^{n} \frac{\left\|x_{1}-p\right\|}{\left\|q_{1}-p\right\|} \\
& \rightarrow 0 \text { as } n \rightarrow \infty
\end{aligned}
$$

Thus, $\left\{x_{n}\right\}$ converges faster than $\left\{q_{n}\right\}$ to $p$, i.e. the Picard-S hybrid iterative process (1.10) converges faster than the S-iterative process:

$$
\text { (ii) } \begin{aligned}
\frac{e_{n}}{b_{n}} & =\frac{\left[\delta^{2}\left(1-(1-\delta) \lambda^{2}\right)\right]^{n}}{\left[\delta\left(1-(1-\delta) \lambda^{2}\right)\right]^{n}} \frac{\left\|x_{1}-p\right\|}{\left\|s_{1}-p\right\|} \\
& =\delta^{n} \frac{\left\|x_{1}-p\right\|}{\left\|s_{1}-p\right\|} \\
& \rightarrow 0 \text { as } n \rightarrow \infty
\end{aligned}
$$

Thus, $\left\{x_{n}\right\}$ converges faster than $\left\{s_{n}\right\}$ to $p$, i.e. the Picard-S hybrid iterative process (1.10) converges faster than the Picard-Mann hybrid iterative process.

$$
\text { (iii) } \begin{aligned}
\frac{e_{n}}{c_{n}} & =\frac{\left[\delta^{2}\left(1-(1-\delta) \lambda^{2}\right)\right]^{n}}{\left[\delta\left(1-(1-\delta) \lambda^{2}\right)\right]^{n}} \frac{\left\|x_{1}-p\right\|}{\left\|m_{1}-p\right\|} \\
& =\delta^{n} \frac{\left\|x_{1}-p\right\|}{\left\|m_{1}-p\right\|} \\
& \rightarrow 0 \text { as } n \rightarrow \infty
\end{aligned}
$$

Thus, $\left\{x_{n}\right\}$ converges faster than $\left\{m_{n}\right\}$ to $p$, i.e. the Picard-S hybrid iterative process (1.10) converges faster than the Picard-Krasnoselskii hybrid iterative process.

$$
\text { (iv) } \begin{aligned}
\frac{e_{n}}{d_{n}} & =\frac{\left[\delta^{2}\left(1-(1-\delta) \lambda^{2}\right)\right]^{n}}{\delta^{n}\left[1-\lambda^{2}(1-\delta)\right]^{n}} \frac{\left\|x_{1}-p\right\|}{\left\|t_{1}-p\right\|} \\
& =\delta^{n} \frac{\left\|x_{1}-p\right\|}{\left\|t_{1}-p\right\|} \\
& \rightarrow 0 \text { as } n \rightarrow \infty
\end{aligned}
$$

Thus, $\left\{x_{n}\right\}$ converges faster than $\left\{t_{n}\right\}$ to $p$, i.e. Picard-S hybrid iterative process converges faster than Picard-Ishikawa hybrid iterative process. This completes the proof of the proposition.

In [8], Okeke proved that the rate of convergence of Picard-Krasnoselskii hybrid iterative process is faster than Picard, Krasnoselskii, Mann and Ishikawa iterations. Agarwal et al. [5] 
proved that S-iteration converges faster than Picard, Krasnoselskii, Mann and Ishikawa iterative processes, and Okeke [9] proved that rate of convergence of Picard-Ishikawa hybrid iterative process is faster than Picard-Mann hibrid and Picard-Krasnoselskii iterations. Therefore, I give an example to show that rate of convergence of Picard-S hybrid iterative process is faster than Picard-Mann hybrid, Picard-Krasnoselskii hybrid and S-iteration. This will show that Picard-S hybrid defined by (1.10) converges to fixed point faster than all other iterations defined in this paper.

Example 3.1. Let $X=R$ and $C=[1,10] \subset X$ and $T: C \rightarrow C$ be an operator defined by $T x=\sqrt[3]{2 x+4}$ for all $x \in C$. Choose $\alpha_{n}=\beta_{n}=\lambda=\frac{1}{2}$ for each $n \in N$ with initial value $x_{1}=5$. For $\delta=\frac{1}{\sqrt[3]{4}}, T$ is a contraction mapping. All the processes converge to the same fixed point 2. It is clear from Table 1 and graphs that our Picard-S hybrid iterative process converges faster than Picard-Ishikawa hybrid, Picard-Mann hybrid, Picard-Krasnoselskii hybrid and S-iteration.

\section{Application to delay differential equation}

Here, I use this new Picard-S hybrid iterative process to find the solution of delay differential equations.

Let $C[a, b]$ be a space of all continuous real valued function on a closed interval $[a, b]$ be endowed with the Chebyshev norm:

$$
\|x-y\|_{\infty}=\max _{t \in[a, b]}|x(t)-y(t)| .
$$

Space $\left(C[a, b],\|\cdot\|_{\infty}\right)$ is known as Banach Space. In this section, the following delay differential equation has been taken:

$$
x^{\prime}(t)=f(t, x(t), x(t-\tau)) \quad t \in[a, b]
$$

with initial condition

$$
x(t)=\phi(t) \quad t \in\left[t_{o}-\tau, t_{o}\right]
$$

By the solution of above problem, we mean a function $x \in C\left(\left[t_{o}-\tau, b\right], R\right) \cap\left(C^{1}\left[t_{o}, b\right], R\right)$

\begin{tabular}{|c|c|c|c|c|c|c|}
\hline Step & Picard-S hybrid & $\begin{array}{c}\text { Picard-Ishikawa } \\
\text { hybrid }\end{array}$ & $\begin{array}{c}\text { Picard-Mann } \\
\text { hybrid }\end{array}$ & $\begin{array}{c}\text { Picard- } \\
\text { Krasnoselskii } \\
\text { hybrid }\end{array}$ & S-iteration & \\
\hline 0 & 5.0000000000000 & 5.0000000000000 & 5.000000000000 & 5.000000000000 & 5.000000000000 & \\
\hline 1 & 2.053665985829 & 2.053665985829 & 2.251284354073 & 2.251284354073 & 2.330713309124 & \\
\hline 2 & 2.001174310362 & 2.001174310362 & 2.024068969098 & 2.024068969098 & 2.042698929425 & \\
\hline 3 & 2.000024999687 & 2.000024999687 & 2.002336639386 & 2.002336639386 & 2.005602850705 & \\
\hline 4 & 2.000000433332 & 2.000000549760 & 2.000227141158 & 2.000227141158 & 2.000738954904 & \\
\hline 5 & 2.000000009450 & 2.000000012089 & 2.000022082864 & 2.000022082864 & 2.000097495596 & \\
\hline 6 & 2.000000000207 & 2.000000000265 & 2.000002146942 & 2.000002146942 & 2.000012863908 & \\
\hline 7 & 2.000000000000 & 2.000000000005 & 2.000000208730 & 2.000000208730 & 2.000001697319 & \\
\hline 8 & & 2.000000000000 & 2.000000020293 & 2.000000020293 & 2.000000223951 & \\
\hline 9 & & & 2.000000001973 & 2.000000001973 & 2.000000029549 & \\
\hline 10 & & & 2.000000000191 & 2.000000000191 & 2.000000003898 & Table 1 \\
\hline 11 & & & 2.000000000018 & 2.000000000018 & 2.000000000514 & A comparison of \\
\hline 12 & & & 2.000000000002 & 2.000000000002 & 2.000000000067 & Picard-S hybrid with \\
\hline 13 & & & 2.000000000000 & 2.000000000000 & 2.000000000008 & other iterative \\
\hline 14 & & & & & 2.000000000000 & processes \\
\hline
\end{tabular}
satisfying (4.1) and (4.2). Assume that the following conditions are satisfied.

$\left(C_{1}\right) t_{o}, b \in R, \tau>0$;
New Picard-S hybrid iteration 


\section{AJMS \\ 28,1}

68
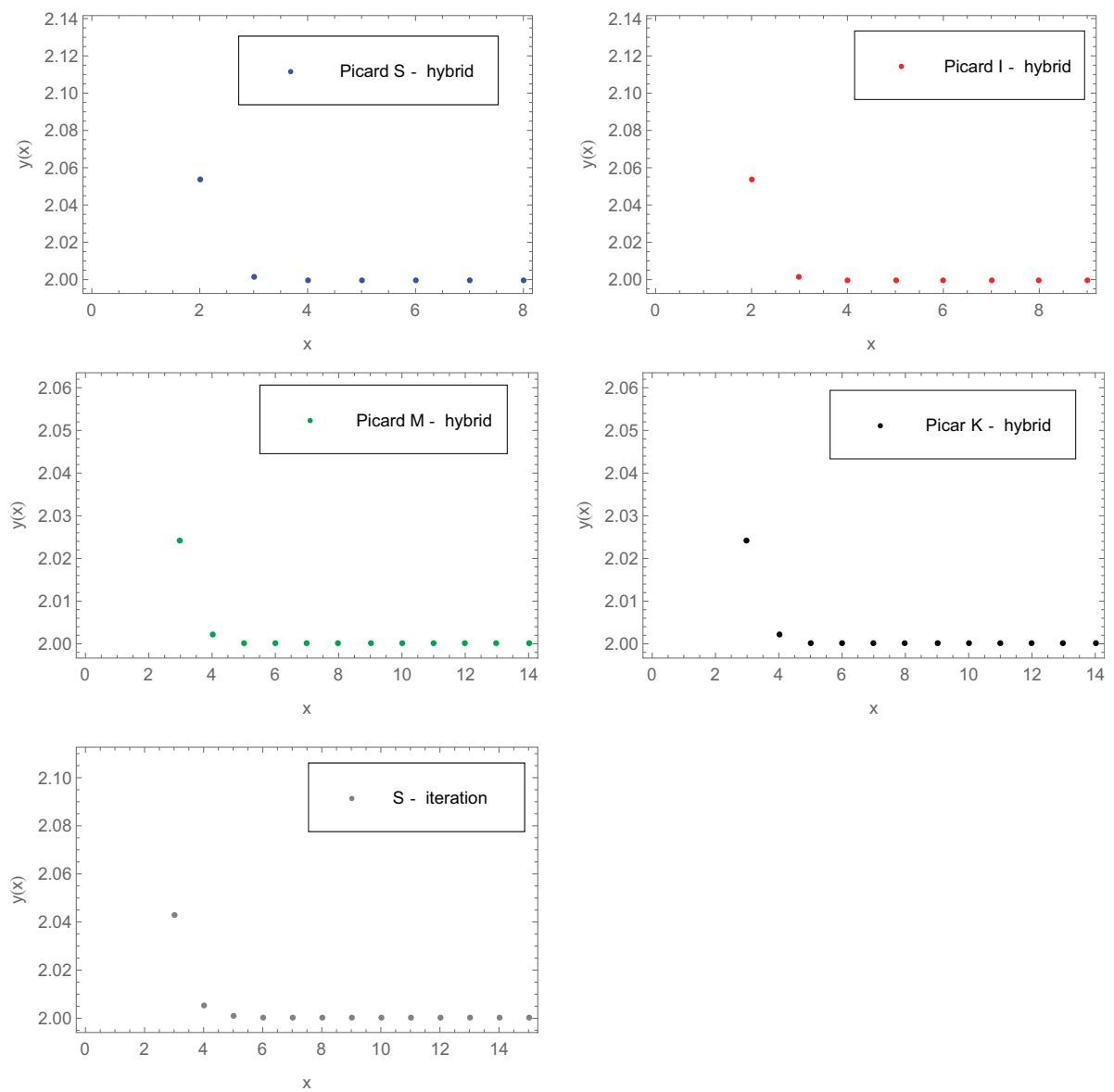

$\left(C_{2}\right) f \in C\left(\left[t_{0}, b\right] \times R^{2}, R\right)$;

$\left(C_{3}\right) \phi \in C\left(\left[t_{0}-\tau, b\right], R\right)$;

$\left(C_{4}\right)$ there exists $L_{f}>0$ such that

$$
\left|f\left(t, u_{1}, u_{2}\right)-f\left(t, v_{1}, v_{2}\right)\right| \leq L_{f} \sum_{i=1}^{2}\left|u_{i}-v_{i}\right| \quad \forall u_{i}, v_{i} \in R, i=1,2 ; t \in\left[t_{o}, b\right]
$$

(C) $2 L_{f}\left(b-t_{o}\right)<1$

Now we can reformulate problems (4.1) and (4.2) by the following integral equation:

$$
x(t)= \begin{cases}\phi(t) & t \in\left[t_{o}-\tau, t_{o}\right] \\ \phi\left(t_{o}\right)+\int_{t_{o}}^{t} f(s, x(s), x(s-\tau)) d s & t \in\left[t_{o}, b\right]\end{cases}
$$

Coman [23] et al. established the following result. 
Theorem 4.1. Assume that the conditions $\left(C_{1}-C_{5}\right)$ are satisfied. Then problem (4.1) with initial condition (4.2) has unique solution $p$ (say) in $C\left(\left[t_{0}-\tau, b\right], R\right) \cap C^{1}\left(\left[t_{0}, b\right], R\right)$ and

$$
p=\lim _{n \rightarrow \infty} T^{n}(x) \quad \text { for any } x \in C\left(\left[t_{0}-\tau, b\right], R\right) .
$$

Using Picard-S hybrid iterative process, I prove the following result.

Theorem 4.2. Assume that $\left(C_{1}\right)-\left(C_{5}\right)$ are satisfied. Then problem (4.1) with initial condition (4.2) has unique solution $p$ (say) in $C\left(\left[t_{o}-\tau, b\right], R\right) \cap C^{1}\left(\left[t_{o}, b\right], R\right)$ and the Picard-S hybrid iterative process (1.10) converges to $p$.

Proof: Let $\left\{x_{n}\right\}$ be an iterative sequence generated by the Picard-S hybrid iterative process (1.10) for an operator defined by

$$
T x(t)= \begin{cases}\phi(t) & t \in\left[t_{o}-\tau, t_{o}\right] \\ \phi\left(t_{o}\right)+\int_{t_{o}}^{t} f(s, x(s), x(s-\tau)) d s & t \in\left[t_{o}, b\right]\end{cases}
$$

Let $p$ be a fixed point of $T$, now I prove that $x_{n} \rightarrow p$ as $n \rightarrow \infty$. It is easy to see that $x_{n} \rightarrow p$ for each $t \in\left[t_{0}-\tau, t_{0}\right]$. Now for each $t \in\left[t_{o}, b\right]$, we have

$$
\begin{aligned}
\left\|x_{n+1}-p\right\|_{\infty} & =\left\|T z_{n}-T p\right\|_{\infty} \\
& =\max _{t \in\left[t_{o}-\tau, b\right]}\left|\int_{t_{o}}^{t} f\left(s, z_{n}(s), z_{n}(s-\tau)\right)-f(s, p(s), p(s-\tau)) d s\right| \\
& \leq \max _{t \in\left[t_{o}-\tau, b\right]} \int_{t_{o}}^{t}\left|f\left(s, z_{n}(s), z_{n}(s-\tau)\right)-f(s, p(s), p(s-\tau))\right| d s \\
& \leq \max _{t \in\left[t_{o}-\tau, b\right]} \int_{t_{o}}^{t} L_{f}\left(\left|z_{n}(s)-p(s)\right|+\left|z_{n}(s-\tau)-p(s-\tau)\right|\right) d s \\
& \leq \int_{t_{o}}^{t} L_{f}\left(\max _{t \in\left[t_{o}-\tau, b\right]}\left|z_{n}(s)-p(s)\right|+\max _{t \in\left[t_{o}-\tau, b\right]}\left|z_{n}(s-\tau)-p(s-\tau)\right|\right) d s \\
& =\int_{t_{o}}^{t} L_{f}\left(\left\|z_{n}-p\right\|_{\infty}+\left\|z_{n}-p\right\|_{\infty}\right) d s \\
& =2\left\|z_{n}-p\right\|_{\infty} \int_{t_{o}}^{t} L_{f} d s \\
& =2\left\|z_{n}-p\right\|_{\infty} L_{f}\left(t-t_{o}\right) \\
& \leq 2 L_{f}\left(b-t_{o}\right)\left\|z_{n}-p\right\|_{\infty}
\end{aligned}
$$

Now,

$$
\begin{aligned}
\left\|z_{n}-p\right\|_{\infty} & =\left\|\left(1-\alpha_{n}\right) T x_{n}+\alpha_{n} T y_{n}-p\right\|_{\infty} \\
& =\left\|\left(1-\alpha_{n}\right)\left(T x_{n}-p\right)+\alpha_{n}\left(T y_{n}-p\right)\right\|_{\infty} \\
& \leq\left(1-\alpha_{n}\right)\left\|T x_{n}-p\right\|_{\infty}+\alpha_{n}\left\|T y_{n}-p\right\|_{\infty}
\end{aligned}
$$

New Picard-S hybrid iteration 
AJMS

28,1

70

$$
\begin{aligned}
\left\|T x_{n}-p\right\|_{\infty} & =\max _{t \in\left[t_{o}-\tau, b\right]}\left|T x_{n}(t)-T p(t)\right| \\
& =\max _{t \in\left[t_{o}-\tau, b\right] \mid}\left|\int_{t_{o}}^{t} f\left(s, x_{n}(s), x_{n}(s-\tau)\right) d s-\int_{t_{o}}^{t} f(s, p(s), p(s-\tau)) d s\right| \\
& \leq \max _{t \in\left[t_{0}-\tau, b\right]} \int_{t_{o}}^{t}\left|f\left(s, x(s), x_{n}(s-\tau)\right)-f(s, p(s), p(s-\tau))\right| d s \\
& =\max _{t \in\left[t_{o}-\tau, b\right]} \int_{t_{o}}^{t} L_{f}\left(\left|x_{n}(s)-p(s)\right|+\left|x_{n}(s-\tau)-p(s-\tau)\right|\right) d s \\
& \leq \int_{t_{o}}^{t} L_{f}\left(\max _{t \in\left[t_{o}-\tau, b\right]}\left|x_{n}(s)-p(s)\right|+\max _{t \in\left[t_{o}-\tau, b\right]}\left|x_{n}(s-\tau)-p(s-\tau)\right|\right) d s \\
& \leq \int_{t_{o}}^{t} L_{f}\left(\left\|x_{n}-p\right\|_{\infty}+\left\|x_{n}-p\right\|_{\infty}\right) d s \\
& =2 L_{f}\left(t-t_{o}\right)\left\|x_{n}-p\right\|_{\infty} \\
& \leq 2 L_{f}\left(b-t_{o}\right)\left\|x_{n}-p\right\|_{\infty}
\end{aligned}
$$

Now,

$$
\begin{aligned}
\left\|y_{n}-p\right\|_{\infty} & =\left\|\left(1-\beta_{n}\right) x_{n}+\beta_{n} T x_{n}-p\right\|_{\infty} \\
& =\left\|\left(1-\beta_{n}\right)\left(x_{n}-p\right)+\beta_{n}\left(T x_{n}-p\right)\right\|_{\infty} \\
& \leq\left(1-\beta_{n}\right)\left\|x_{n}-p\right\|_{\infty}+\beta_{n}\left\|T x_{n}-p\right\|_{\infty}
\end{aligned}
$$

Now,

$$
\begin{aligned}
\left\|T y_{n}-p\right\|_{\infty} & =\left\|T y_{n}-T p\right\|_{\infty} \\
& =\max _{t \in\left[t_{o}-\tau, b\right]}\left|\int_{t_{o}}^{t} f\left(s, y_{n}(s), y_{n}(s-\tau)\right) d s-\int_{t_{o}}^{t} f(s, p(s), p(s-\tau)) d s\right| \\
& \leq \max _{t \in\left[t_{o}-\tau, b\right]} \int_{t_{o}}^{t}\left|f\left(s, y_{n}(s), y_{n}(s-\tau)\right)-f(s, p(s), p(s-\tau))\right| d s \\
& \leq \max _{t \in\left[t_{o}-\tau, b\right]} \int_{t_{o}}^{t} L_{f}\left(\left|y_{n}(s)-p(s)\right|+\left|y_{n}(s-\tau)-p(s-\tau)\right|\right) d s \\
& \leq \int_{t_{o}}^{t} L_{f}\left(\max _{t \in\left[t_{o}-\tau, b\right]}\left|y_{n}(s)-p(s)\right|+\max _{t \in\left[t_{0}-\tau, b\right]}\left|y_{n}(s-\tau)-p(s-\tau)\right|\right) d s \\
& =\int_{t_{o}}^{t} L_{f}\left(\left\|y_{n}-p\right\|_{\infty}+\left\|y_{n}-p\right\|_{\infty}\right) d s \\
& =2\left\|y_{n}-p\right\|_{\infty} \int_{t_{o}}^{t} L_{f} d s \\
& =2\left\|y_{n}-p\right\|_{\infty} L_{f}\left(t-t_{o}\right) \\
& \leq 2 L_{f}\left(b-t_{o}\right)\left\|y_{n}-p\right\|_{\infty}
\end{aligned}
$$


Using (4.6) in (4.7), we get

$$
\begin{aligned}
\left\|T y_{n}-p\right\|_{\infty} & =\left\|T y_{n}-T p\right\|_{\infty} \\
& \leq 2 L_{f}\left(b-t_{0}\right)\left\{\left(1-\beta_{n}\right)\left\|x_{n}-p\right\|_{\infty}+\beta_{n}\left\|T x_{n}-p\right\|_{\infty}\right\} \\
& =2\left(1-\beta_{n}\right) L_{f}\left(b-t_{o}\right)\left\|x_{n}-p\right\|_{\infty}+2 \beta_{n} L_{f}\left(b-t_{o}\right)\left\|T x_{n}-p\right\|_{\infty}
\end{aligned}
$$

New Picard-S hybrid iteration

From (4.5) we get

$$
\begin{aligned}
\left\|T y_{n}-p\right\|_{\infty} & \leq 2\left(1-\beta_{n}\right) L_{f}\left(b-t_{o}\right)\left\|x_{n}-p\right\|_{\infty}+2 \beta_{n} L_{f}\left(b-t_{o}\right) 2 L_{f}\left(b-t_{o}\right)\left\|x_{n}-p\right\|_{\infty} \\
& =2 L_{f}\left(b-t_{o}\right)\left\{\left(1-\beta_{n}\right)+2 \beta_{n} L_{f}\left(b-t_{o}\right)\right\}\left\|x_{n}-p\right\|_{\infty}
\end{aligned}
$$

Using (4.5) and (4.9) in (4.4), we get

$$
\begin{aligned}
\left\|z_{n}-p\right\|_{\infty} & \leq\left(1-\alpha_{n}\right) 2 L_{f}\left(b-t_{o}\right)\left\|x_{n}-p\right\|_{\infty}+\alpha_{n}\left[2 L_{f}\left(b-t_{0}\right)\left\{\left(1-\beta_{n}\right)+2 \beta_{n} L_{f}\left(b-t_{o}\right)\right\}\left\|x_{n}-p\right\|_{\infty}\right] \\
& =2 L_{f}\left(b-t_{o}\right)\left[\left(1-\alpha_{n}\right)+\alpha_{n}\left(1-\beta_{n}\right)+2 \alpha_{n} \beta_{n} L_{f}\left(b-t_{o}\right)\right]\left\|x_{n}-p\right\|_{\infty} \\
& =2 L_{f}\left(b-t_{o}\right)\left[1-\alpha_{n} \beta_{n}+2 \alpha_{n} \beta_{n} L_{f}\left(t-t_{o}\right)\right]\left\|x_{n}-p\right\|_{\infty}
\end{aligned}
$$

Note that $2 L_{f}\left(b-t_{0}\right)\left[1-\alpha_{n} \beta_{n}+2 \alpha_{n} \beta_{n} L_{f}\left(t-t_{o}\right)\right]=\mu_{n}<1$ and $\left\|x_{n}-P\right\|_{\infty}=S_{n}$. Thus, all conditions of lemma 2.1 are satisfied. Hence, $\lim _{n \rightarrow \infty}\left\|x_{n}-P\right\|_{\infty}=0$. This completes the proof of above theorem.

\section{Picard-S hybrid iterative process for nonexpansive mappings}

Lemma 5.1. Let $E$ be a normed space, $C$ a non-empty convex subset of $E$ and $T: C \rightarrow C$ a nonexpansive mapping. If $\left\{x_{n}\right\}$ is the iterative process defined by (1.10), then $\lim _{n \rightarrow \infty}\left\|x_{n}-T x_{n}\right\|$ exists.

Proof: Set $a_{n}=x_{n}-T x_{n}$ for all $n \in N$. Then we have

$$
\begin{aligned}
\left\|a_{n+1}\right\| & =\left\|x_{n+1}-T x_{n+1}\right\| \\
& =\left\|T z_{n}-T x_{n+1}\right\| \\
& \leq\left\|z_{n}-x_{n+1}\right\| \\
& =\left\|\left(1-\alpha_{n}\right) T x_{n}+\alpha_{n} T y_{n}-T z_{n}\right\| \\
& =\left\|\left\{\left(1-\alpha_{n}\right)\left(T x_{n}-T z_{n}\right)\right\}+\left\{\alpha_{n}\left(T y_{n}-T z_{n}\right)\right\}\right\| \\
& \leq\left(1-\alpha_{n}\right)\left\|T x_{n}-T z_{n}\right\|+\alpha_{n}\left\|T y_{n}-T z_{n}\right\| \\
& \leq\left(1-\alpha_{n}\right)\left\|x_{n}-z_{n}\right\|+\alpha_{n}\left\|y_{n}-z_{n}\right\| \\
\left\|x_{n}-z_{n}\right\| & =\left\|x_{n}-\left\{\left(1-\alpha_{n}\right) T x_{n}+\alpha_{n} T y_{n}\right\}\right\| \\
& =\left\|\left(x_{n}-T x_{n}\right)+\alpha_{n}\left(T x_{n}-T y_{n}\right)\right\| \\
& \leq\left\|x_{n}-T x_{n}\right\|+\alpha_{n}\left\|T x_{n}-T y_{n}\right\| \\
& \leq\left\|x_{n}-T x_{n}\right\|+\alpha_{n}\left\|x_{n}-y_{n}\right\| \\
& =\left\|a_{n}\right\|+\alpha_{n}\left\|x_{n}-y_{n}\right\|
\end{aligned}
$$

Now,

$$
\begin{aligned}
\left\|x_{n}-y_{n}\right\| & =\left\|x_{n}-\left\{\left(1-\beta_{n}\right) x_{n}+\beta_{n} T x_{n}\right\}\right\| \\
& =\left\|x_{n}-x_{n}+\beta_{n} x_{n}-\beta_{n} T x_{n}\right\| \\
& =\beta_{n}\left\|x_{n}-T x_{n}\right\| \\
& =\beta_{n}\left\|a_{n}\right\|
\end{aligned}
$$


AJMS

28,1

From inequality (5.2) and (5.3), we have

$$
\begin{aligned}
\left\|x_{n}-z_{n}\right\| & \leq\left\|a_{n}\right\|+\alpha_{n} \beta_{n}\left\|\alpha_{n}\right\| \\
& =\left(1+\alpha_{n} \beta_{n}\right)\left\|\alpha_{n}\right\|
\end{aligned}
$$

Now,

$$
\begin{aligned}
\left\|y_{n}-z_{n}\right\| & =\left\|y_{n}-\left\{\left(1-\alpha_{n}\right) T x_{n}+\alpha_{n} T y_{n}\right\}\right\| \\
& =\left\|\left(1-\alpha_{n}\right) y_{n}+\alpha_{n} y_{n}-\left(1-\alpha_{n}\right) T x_{n}-\alpha_{n} T y_{n}\right\| \\
& =\left\|\left(1-\alpha_{n}\right)\left(y_{n}-T x_{n}\right)+\alpha_{n}\left(y_{n}-T y_{n}\right)\right\| \\
& \leq\left(1-\alpha_{n}\right)\left\|y_{n}-T x_{n}\right\|+\alpha_{n}\left\|y_{n}-T y_{n}\right\|
\end{aligned}
$$

Now,

$$
\begin{aligned}
\left\|y_{n}-T x_{n}\right\| & =\left\|\left(1-\beta_{n}\right) x_{n}+\beta_{n} T x_{n}-T x_{n}\right\| \\
& =\left\|\left(1-\beta_{n}\right) x_{n}-\left(1-\beta_{n}\right) T x_{n}\right\| \\
& \leq\left(1-\beta_{n}\right)\left\|x_{n}-T x_{n}\right\| \\
& =\left(1-\beta_{n}\right)\left\|a_{n}\right\| \\
\left\|y_{n}-T y_{n}\right\| & =\left\|\left(1-\beta_{n}\right) x_{n}+\beta_{n} T x_{n}-T y_{n}\right\| \\
& =\left\|\left(1-\beta_{n}\right) x_{n}+\beta_{n} T x_{n}-\left(1-\beta_{n}+\beta_{n}\right) T y_{n}\right\| \\
& =\left\|\left(1-\beta_{n}\right)\left(x_{n}-T y_{n}\right)+\beta_{n}\left(T x_{n}-T y_{n}\right)\right\| \\
& \leq\left(1-\beta_{n}\right)\left\|x_{n}-T y_{n}\right\|+\beta_{n}\left\|T x_{n}-T y_{n}\right\| \\
& =\left(1-\beta_{n}\right)\left\|x_{n}-T y_{n}\right\|+\beta_{n}\left\|x_{n}-y_{n}\right\|
\end{aligned}
$$

Using (5.3) in (5.7), we get

$$
\begin{aligned}
\left\|y_{n}-T y_{n}\right\| & \leq\left(1-\beta_{n}\right)\left\|x_{n}-T y_{n}\right\|+\beta_{n} \beta_{n}\left\|a_{n}\right\| \\
& =\left(1-\beta_{n}\right)\left\|x_{n}-T y_{n}\right\|+\beta_{n}^{2}\left\|a_{n}\right\|
\end{aligned}
$$

Using (5.6) and (5.8) in (5.5), we get

$$
\begin{aligned}
\left\|y_{n}-z_{n}\right\| & \leq\left(1-\alpha_{n}\right)\left(1-\beta_{n}\right)\left\|a_{n}\right\|+\alpha_{n}\left\{\left(1-\beta_{n}\right)\left\|x_{n}-T y_{n}\right\|+\beta_{n}^{2}\left\|a_{n}\right\|\right\} \\
& =\left(1-\alpha_{n}\right)\left(1-\beta_{n}\right)\left\|a_{n}\right\|+\alpha_{n}\left\{\left(1-\beta_{n}\right)\left\|x_{n}-T x_{n}+T x_{n}-T y_{n}\right\|+\beta_{n}^{2}\left\|a_{n}\right\|\right\} \\
& \leq\left(1-\alpha_{n}\right)\left(1-\beta_{n}\right)\left\|a_{n}\right\|+\alpha_{n}\left\{\left(1-\beta_{n}\right)\left(\left\|x_{n}-T x_{n}\right\|+\left\|T x_{n}-T y_{n}\right\|\right)+\beta_{n}^{2}\left\|a_{n}\right\|\right\} \\
& \leq\left(1-\alpha_{n}\right)\left(1-\beta_{n}\right)\left\|a_{n}\right\|+\alpha_{n}\left\{\left(\left(1-\beta_{n}\right)\left\|x_{n}-T x_{n}\right\|+\left(1-\beta_{n}\right)\left\|x_{n}-y_{n}\right\|\right)+\beta_{n}^{2}\left\|a_{n}\right\|\right\} \\
& =\left(1-\alpha_{n}\right)\left(1-\beta_{n}\right)\left\|a_{n}\right\|+\alpha_{n}\left\{\left(\left(1-\beta_{n}\right)\left\|a_{n}\right\|+\left(1-\beta_{n}\right)\left\|x_{n}-y_{n}\right\|\right)+\beta_{n}^{2}\left\|a_{n}\right\|\right\}
\end{aligned}
$$

Using (5.3) in (5.9), we get

$$
\begin{aligned}
\left\|y_{n}-z_{n}\right\| & \leq\left(1-\alpha_{n}\right)\left(1-\beta_{n}\right)\left\|a_{n}\right\|+\alpha_{n}\left\{\left(\left(1-\beta_{n}\right)\left\|a_{n}\right\|+\left(1-\beta_{n}\right) \beta_{n}\left\|a_{n}\right\|\right)+\beta_{n}^{2}\left\|a_{n}\right\|\right\} \\
& =\left[\left(1-\alpha_{n}\right)\left(1-\beta_{n}\right)+\alpha_{n}\left(1-\beta_{n}\right)\left(1+\beta_{n}\right)+\alpha_{n} \beta_{n}^{2}\right]\left\|a_{n}\right\| \\
& =\left(1-\beta_{n}+\alpha_{n} \beta_{n}\right)\left\|a_{n}\right\|
\end{aligned}
$$

From (5.1), (5.4) and (5.10), we get

$$
\begin{aligned}
\left\|a_{n+1}\right\| & \leq\left[\left(1-\alpha_{n}\right)\left(1+\alpha_{n} \beta_{n}\right)\right]\left\|a_{n}\right\|+\left[\alpha_{n}\left(1-\beta_{n}+\alpha_{n} \beta_{n}\right)\right]\left\|a_{n}\right\| \\
& =\left[\left(1-\alpha_{n}\right)\left(1+\alpha_{n} \beta_{n}\right)+\alpha_{n}\left(1-\beta_{n}+\alpha_{n} \beta_{n}\right)\right]\left\|a_{n}\right\| \\
& =\left[1+\alpha_{n} \beta_{n}-\alpha_{n}-\alpha_{n}^{2} \beta_{n}+\alpha_{n}-\alpha_{n} \beta_{n}+\alpha_{n}^{2} \beta_{n}\right]\left\|a_{n}\right\| \\
& =\left\|a_{n}\right\|
\end{aligned}
$$

So that $\left\{\left\|a_{n}\right\|\right\}$ is nonincreasing and hence, $\lim _{n \rightarrow \infty}\left\|a_{n}\right\|$ exists. 
Theorem 5.2. [5] Let $X$ be a Banach space with modulus of convexity $\delta_{X}$. Then

$$
\|(1-t) x+t y\| \leq 1-2 t(1-t) \delta_{X}(\|x-y\|)
$$

for all $x, y \in X$ with $\|x\| \leq 1,\|y\| \leq 1$ and all $t \in[0,1]$.

Theorem 5.3. Let $C$ be a non-empty closed convex (not necessary bounded) subset of a uniformly convex Banach space $X$ and $T: C \rightarrow C$ a nonexpansive mapping. Let $\left\{x_{n}\right\}$ be the sequence defined by (1.10) with the restriction:

$$
\lim _{n \rightarrow \infty} \alpha_{n} \beta_{n}\left(1-\alpha_{n}\right) \text { exists and } \lim _{n \rightarrow \infty} \alpha_{n} \beta_{n}\left(1-\beta_{n}\right) \neq 0
$$

Then, for arbitrary initial value $x_{1} \in C,\left\{\left\|x_{n}-T x_{n}\right\|\right\}$ converges to some constant $\gamma_{C}(T)=\inf \{\|x-T x\|: x \in C\}$, which is independent of the choice of the initial value $x_{1} \in C$.

Proof: Lemma (5.1) implies that $\lim \left\|x_{n}-T x_{n}\right\|$ exists and denote $\gamma\left(x_{1}\right)=\lim \left\|x_{n}-T x_{n}\right\|$. Let $\left\{x_{n}^{*}\right\}$ be another iterative sequence generated by (1.10) with the same re restriction on parameters $\left\{\alpha_{n}\right\}$ and $\left\{\beta_{n}\right\}$ of iteration as the sequence $\left\{x_{n}\right\}$ but with the initial value $x_{1}^{*} \in C$. It follows from lemma (5.1) that

$$
\lim _{n \rightarrow \infty}\left\|x_{n}^{*}-T x_{n}^{*}\right\|=\gamma\left(x_{1}^{*}\right)
$$

Observe that

$$
\begin{aligned}
\left\|T y_{n}-T y_{n}^{*}\right\| & \leq\left\|y_{n}-y_{n}^{*}\right\| \\
& \leq\left(1-\beta_{n}\right)\left\|x_{n}-x_{n}^{*}\right\|+\beta_{n}\left\|T x_{n}-T x_{n}^{*}\right\| \\
& \leq\left(1-\beta_{n}\right)\left\|x_{n}-x_{n}^{*}\right\|+\beta_{n}\left\|x_{n}-x_{n}^{*}\right\| \\
& \leq\left\|x_{n}-x_{n}^{*}\right\|
\end{aligned}
$$

Now

$$
\begin{aligned}
\left\|x_{n+1}-x_{n+1}^{*}\right\| & =\left\|T z_{n}-T z_{n}^{*}\right\| \\
& \leq\left\|z_{n}-z_{n}^{*}\right\| \\
& =\left\|\left(1-\alpha_{n}\right)\left(T x_{n}-T x_{n}^{*}\right)+\alpha_{n}\left(T y_{n}-T y_{n}^{*}\right)\right\| \\
& \leq\left(1-\alpha_{n}\right)\left\|T x_{n}-T x_{n}^{*}\right\|+\alpha_{n}\left\|T y_{n}-T y_{n}^{*}\right\| \\
& \leq\left(1-\alpha_{n}\right)\left\|x_{n}-x_{n}^{*}\right\|+\alpha_{n}\left\|T y_{n}-T y_{n}^{*}\right\|
\end{aligned}
$$

Using (5.12) in (5.13), we have

$$
\begin{aligned}
\left\|x_{n+1}-x_{n+1}^{*}\right\| & \leq\left(1-\alpha_{n}\right)\left\|x_{n}-x_{n}^{*}\right\|+\alpha_{n}\left\|x_{n}-x_{n}^{*}\right\| \\
& =\left\|x_{n}-x_{n}^{*}\right\|
\end{aligned}
$$

This shows that $\lim _{n \rightarrow \infty}\left\|x_{n}-x_{n}^{*}\right\|$ exists.

Let $\lim _{n \rightarrow \infty}\left\|x_{n}-x_{n}^{*}\right\|=d$ for some $d>0$.

Let

$$
\begin{aligned}
\left\|y_{n}-y_{n}^{*}\right\| & =\left\|\left(1-\beta_{n}\right)\left(x_{n}-x_{n}^{*}\right)+\beta_{n}\left(T x_{n}-T x_{n}^{*}\right)\right\| \\
& =\left\|\frac{\left(1-\beta_{n}\right)\left(x_{n}-x_{n}^{*}\right)}{\left\|x_{n}-x_{n}^{*}\right\|}+\frac{\beta_{n}\left(T x_{n}-T x_{n}^{*}\right)}{\left\|x_{n}-x_{n}^{*}\right\|}\right\|\left\|x_{n}-x_{n}^{*}\right\|
\end{aligned}
$$


AJMS

28,1

74
Since

$$
\begin{gathered}
\left\|\frac{\left(x_{n}-x_{n}^{*}\right)}{\left\|x_{n}-x_{n}^{*}\right\|}\right\|=\frac{\left\|x_{n}-x_{n}^{*}\right\|}{\left\|x_{n}-x_{n}^{*}\right\|}=1 \\
\left\|\frac{\left(T x_{n}-T x_{n}^{*}\right)}{\left\|x_{n}-x_{n}^{*}\right\|}\right\|=\frac{\left\|T x_{n}-T x_{n}^{*}\right\|}{\left\|x_{n}-x_{n}^{*}\right\|} \leq \frac{\left\|x_{n}-x_{n}^{*}\right\|}{\left\|x_{n}-x_{n}^{*}\right\|}=1
\end{gathered}
$$

Using theorem (5.2) and (5.16), we obtain that

$$
\left\|y_{n}-y_{n}^{*}\right\| \leq 1-2 \beta_{n}\left(1-\beta_{n}\right) \delta_{X}\left(\frac{\left\|x_{n}-x_{n}^{*}-\left(T x_{n}-T x_{n}^{*}\right)\right\|}{\left\|x_{n}-x_{n}^{*}\right\|}\right)\left\|x_{n}-x_{n}^{*}\right\|
$$

It follows from (5.14) that

$$
\begin{aligned}
\left\|x_{n+1}-x_{n+1}^{*}\right\| & \leq\left(1-\alpha_{n}\right)\left\|x_{n}-x_{n}^{*}\right\|+\alpha_{n}\left\|x_{n}-x_{n}^{*}\right\|\left[1-2 \beta_{n}\left(1-\beta_{n}\right) \delta_{X}\left(\frac{\left\|\left(x_{n}-x_{n}^{*}\right)-\left(T x_{n}-T x_{n}^{*}\right)\right\|}{\left\|x_{n}-x_{n}^{*}\right\|}\right)\right] \\
& =\left\|x_{n}-x_{n}^{*}\right\|-2 \alpha_{n} \beta_{n}\left(1-\beta_{n}\right)\left\|x_{n}-x_{n}^{*}\right\| \delta_{X}\left(\frac{\left\|\left(x_{n}-x_{n}^{*}\right)-\left(T x_{n}-T x_{n}^{*}\right)\right\|}{\left\|x_{1}-x_{1}^{*}\right\|}\right)
\end{aligned}
$$

This gives us

$$
2 \alpha_{n} \beta_{n}\left(1-\beta_{n}\right)\left\|x_{n}-x_{n}^{*}\right\| \delta_{X}\left(\frac{\left\|\left(x_{n}-x_{n}^{*}\right)-\left(T x_{n}-T x_{n}^{*}\right)\right\|}{\left\|x_{n}-x_{n}^{*}\right\|}\right) \leq\left\|x_{n}-x_{n}^{*}\right\|-\left\|x_{n+1}-x_{n+1}^{*}\right\|
$$

Or

$$
2 \alpha_{n} \beta_{n}\left(1-\beta_{n}\right)\left\|x_{n}-x_{n}^{*}\right\| \delta_{X}\left(\frac{\left\|\left(x_{n}-x_{n}^{*}\right)-\left(T x_{n}-T x_{n}^{*}\right)\right\|}{\left\|x_{n}-x_{n}^{*}\right\|}\right) \leq\left\|x_{1}-x_{1}^{*}\right\|
$$

Using restriction $\lim _{n \rightarrow \infty} \alpha_{n} \beta_{n}\left(1-\beta_{n}\right) \neq 0$ and $\lim _{n \rightarrow \infty}\left\|x_{n}-x_{n}^{*}\right\|=d>0$.
Therefore,

$$
\lim _{n \rightarrow \infty} \delta_{X}\left(\frac{\left\|\left(x_{n}-x_{n}^{*}\right)-\left(T x_{n}-T x_{n}^{*}\right)\right\|}{\left\|x_{n}-x_{n}^{*}\right\|}\right)=0
$$

$\delta_{X}$ is strictly increasing and continuous and $\lim _{n \rightarrow \infty}\left\|x_{n}-x_{n}^{*}\right\|=d>0$.
We have

$$
\lim _{n \rightarrow \infty}\left\|\left(x_{n}-x_{n}^{*}\right)-\left(T x_{n}-T x_{n}^{*}\right)\right\|=0
$$

Observe that

$$
\left|\left\|x_{n}-T x_{n}\right\|-\left\|x_{n}^{*}-T x_{n}^{*}\right\|\right| \leq\left\|\left(x_{n}-T x_{n}\right)-\left(x_{n}^{*}-T x_{n}^{*}\right)\right\|
$$

which implies that

$$
\lim _{n \rightarrow \infty}\left|\left\|x_{n}-T x_{n}\right\|-\left\|x_{n}^{*}-T x_{n}^{*}\right\|\right|=0
$$


Thus, $\gamma\left(x_{1}\right)=\gamma\left(x_{1}^{*}\right)$. Because

$$
\left\|x_{n+1}-T x_{n+1}\right\| \leq\left\|x_{n}-T x_{n}\right\| \leq\left\|x_{1}-T x_{1}\right\|
$$

for all $n \in N$ and $x_{1} \in C$

It follows that

$$
\gamma_{C}(T)=\inf \{\|x-T x\|: x \in C\}
$$

\section{References}

[1] Picard E. Memoire surla theorie des equations aux derivees partielles et la methode des approximations successives. J Math Pures Appl. 1890; 6: 145-210.

[2] Krasnoselskii, MA. Two observations about the method of successive approximation. Usp Mat Nauk. 1955; 10: 123-27.

[3] Mann WR. Mean value methods in iteration. Proc Am Math Soc. 1953; 4: 506-70.

[4] Ishikawa S. Fixed points by a new iteration method. Proc Am Math Soc. 1974; 44: 147-50.

[5] Agarwal RP, O'Regan D and Sahu DR. Fixed point theory for lipschitzion-type mappings with applications. New York: Springer Dordrecht Heidelberg London; 2009.

[6] Chidume $\mathrm{CE}$ and Olaleru JO. Picard iteration process for a general class of contractive mappings. J Niger Math Soc. 2014; 33: 19-23.

[7] Khan SH and Picard-Mann A. Hybrid iterative process. Fixed Point Theory Appl. 2013; Article number: 69(2013).

[8] Okeke GA and Abbas M. A solution of delay differential equation via Picard-Krasnoselskii hybrid iterative process. Arab J Math. 2017; 6: 21-9. doi: 10.1007/540065-017-0162-8.

[9] Okeke GA. Convergence analysis of the Picard-Ishikawa hybrid iterative process with applications. Afrika Mathematica 2019; 30: 817-35.

[10] Rhoades BE. Comments on two fixed point iteration methods. J Math Anal Appl. 1976; 56(3): 741-50.

[11] Abbas M and Nazir T. A new faster iteration process applied to constrained minimization and feasibility problems. Mat Vesn. 2014; 66(2): 223-34.

[12] Babu GVR and Vara Prasad KNV. Mann iteration converges faster than Ishikawa iteration for the class of Zamfirescu operators. Fixed Point Theory Appl. 2006; 6, 49615.

[13] Berinde V. Picard iteration converges faster than Mann iteration for class of quasi contractive operators. Fixed Point Theory Appl. 2004; 2004, 716359.

[14] Berinde V and Berinde M. The fastest Krasnoselskii iteration for approximating fixed points of strictly pseudo contractive mappings. Carpathian J Math. 2005; 21(1-2): 13-20.

[15] Berinde V and Pacurar M. Empirical study of the rate of convergence of some fixed point iterative methods. Proc Appl Math Mech. 2007; 7, 2030015-16. doi 10.1002/pamm.200700254.

[16] Dogan K and Karakaya V. On the convergence and stability results for a new general iterative process. Sci World J. 2014; 8, 852475.

[17] Gursoy F, Karakaya V and Picard SA. Hybrid type iteration method for solving a differential equation with retarded arguments. arXiv:1403.2546v2 [math FA], 28 April 2014.

[18] Rhoades BE and Xue Z. Comparison of the rate of convergence among Picard, Mann, Ishikawa, and Noor iterations applied to quasicontractive maps. Fixed Point Theory Appl. 2010; $12,169062$.

[19] Xue Z. The comparison of the convergence speed between Picard, Mann, Krasnoselskii and Ishikawa iterations in Banach spaces. Fixed Point Theory Appl. 2008; 5, 387056. 
AJMS

28,1

76

[20] Berinde V. Iterative approximation of fixed points, Baia Mare: Efemeride; 2002.

[21] Zhao HY. Pseudo almost periodic solutions for a class of differential equation with delays depending on state. Adv Nonlinear Anal. 2020; 9: 1251-8.

[22] Sahin A. Some results of the Picard-Krasnoselskii hybrid process. Filomat. 2019; 33(2): 359-65.

[23] Coman GH, Pavel G, Rus I and Rus IA. Introduction in the theory of operational equation, Cluj-Napoca: Ed. Dacia; 1976.

\section{Further reading}

[24] Nelson PW, Murray JD and Perelson AS. A model of HIV-1 pathogenesis that includes an intracellular delay, Math Biosci. 2000; 163: 201-15.

[25] Soltuz SM and Otrocol D. Classical results via Mann-Ishikawa iteration. Revue d'Analyse Numerique et de Theorie de I'Approximation. 2007; 36(2): 195-9.

\section{Corresponding author}

Julee Srivastava can be contacted at: mathjulee@gmail.com

For instructions on how to order reprints of this article, please visit our website:

www.emeraldgrouppublishing.com/licensing/reprints.htm

Or contact us for further details: permissions@emeraldinsight.com 